\title{
Risk-reducing surgery for ovarian cancer: outcomes in 300 surgeries suggest a low peritoneal primary risk
}

\author{
D Gareth R Evans ${ }^{*, 1}$, Richard Clayton ${ }^{2}$, Paul Donnai ${ }^{2}$, Andrew Shenton ${ }^{1}$ and Fiona Lalloo ${ }^{1}$
}

\begin{abstract}
${ }^{1}$ Medical Genetics Research Group and Regional Genetics Service, University of Manchester and Central Manchester and Manchester Children's University Hospitals NHS Trust, St Mary's Hospital, Manchester, UK; ${ }^{2}$ Gynaecology Department, University of Manchester and Central Manchester and Manchester Children's University Hospitals NHS Trust, St Mary's Hospital, Manchester, UK
\end{abstract}

Risk-reducing salpingo-oophorectomy is currently advocated for the reduction of both breast and ovarian cancer risk in $B R C A 1 / 2$ carriers, but residual risk of peritoneal primary cancer remains a concern. A sequential series of women attending a single institution for ovarian risk management underwent either risk-reducing surgery or screening. A person-years at risk analysis was used to compare observed versus expected cancers. In total, 300 women underwent risk-reducing salpingo-oophorectomy, including 160 $B R C A 1 / 2$ mutation carriers. Three occult ovarian cancers were detected at surgery. There have been 2400.4 years of follow-up and 15.79 expected cancers. No peritoneal cancers have occurred. Amongst 503 women controls with 3444.3 years of follow-up, 15.93 ovarian cancers were expected and 17 were found. There were six ovarian cancer-related deaths in the control group compared with one in the surgery group. Risk-reducing salpingo-oophorectomy in a single institution has so far avoided peritoneal cancer incidence. European Journal of Human Genetics (2009) 17, 1381-1385; doi:10.1038/ejhg.2009.60; published online 15 April 2009

Keywords: BRCA1; BRCA2; risk reducing oophorectomy; prophylactic; ovarian cancer

\section{Introduction}

Ovarian cancer is the most common gynaecological cancer, affecting $\sim 1$ in 70 of the general female population. Ovarian cancer generally presents late, and the overall 5 -year survival for ovarian cancer is only $28 \%$. However, if diagnosed at stages $1-2$, the prognosis is much improved with a 5 -year survival of up to $84 \% .^{1}$

Epithelial tumours account for over $90 \%$ of all ovarian cancers and the lifetime risk is estimated to be between 1 and $1.5 \%$ for the average woman. This risk increases with the number of affected relatives with ovarian cancer and

*Correspondence: Professor DGR Evans, Medical Genetics Research Group and Regional Genetics Service, University of Manchester and Central Manchester and Manchester Children's University Hospitals NHS Trust,

St Mary's Hospital, Manchester M13 0JH, UK. Tel: +1 161 2766206;

Fax: + 1161 2766145; E-mail: Gareth.evans@cmmc.nhs.uk

Received 10 November 2008; revised 23 February 2009; accepted 12 March 2009; published online 15 April 2009 the closeness of the relationship. The lifetime risk estimate for women with one first degree relative is $2-5 \%$ and when there is more than one close relative affected, the risk increases to $10-23 \% .^{2-4}$ Highly penetrant cancerpredisposing genes account for $8-13 \%$ of all epithelial ovarian cancers in non-founder populations, ${ }^{3-6}$ but this increases to $23 \%$ in Norway, and is even higher in the Jewish population. ${ }^{7}$ Those identified to date include $B R C A 1$ and BRCA2, associated with breast and ovarian cancer, and the mismatch repair genes associated with Hereditary Non-Polyposis Colon Cancer (HNPCC). Germline mutations in these two classes of genes can confer an increased risk of ovarian cancer of up to 60 and $12 \%$, respectively. ${ }^{8-10}$ In most instances, increased genetic risk is initially recognised from family history, and mutation screening may then become available. For women at increased risk, management options include ovarian screening and prophylactic surgery. ${ }^{3}$ Demand for ovarian cancer screening has risen, yet there is no clear evidence of 
its effectiveness in groups at increased risk. ${ }^{10-14}$ Indeed, survival in screened individuals with BRCA1/2 mutations is little different to what might be expected without screening, although there may be a benefit in Lynch syndrome. ${ }^{14}$ Despite the paucity of evidence of efficacy of ovarian screening, many reviews remain upbeat regarding the potential outcomes of surveillance. ${ }^{15}$ In contrast, the efficacy of risk-reducing surgery is proven with a risk reduction of $79-95 \% .^{16-18}$ However, there is still a concern regarding the risk of peritoneal primary malignancy ${ }^{16-19}$ in high-risk families. We have reviewed our experience at a single gynaecology centre offering riskreducing surgery.

\section{Patients and methods}

Women attending the cancer genetic clinic at St Mary's Hospital had their risk of ovarian cancer evaluated from empiric epidemiological data or from estimates of the likelihood of a $B R C A 1 / B R C A 2$-associated risk. ${ }^{2,4}$ Lifetime risks of between 3 and $60 \%$ were derived. To assess risk over time, it was assumed that the inherited risk remained constant between 40 and 80 years, and that there was no risk before 40 years except in HNPCC and BRCA1 mutation carriers. BRCA1 and HNPCC carriers were assumed to have a $3 \%$ risk before 40 years of age with the remainder of their risk distributed equally from 40 to 80 years. Follow-up was considered from the date of risk-reducing surgery to last known follow-up, death or 01/03/2008. All patients were checked against the North West Cancer Intelligence Service (NWCIS) database on 01/09/2008 for any malignancy, of which we may have been unaware. This follow-up period allowed for a 6-month lag in notification to the NWCIS. A person-years at risk analysis was carried out from the date of surgery for bilateral salpingo-oophorectomy (BSO) or date of prevalence scan for the control group, to date of last follow-up relevant to the group (women choosing surgery were excluded at date of surgery). Expected ovarian cancers for each individual were summated. No adjustments were made for hormonal or reproductive risk factors.

All ovarian surgeries were carried out with a strict protocol involving immediate bagging of the ovaries and peritoneal lavage to reduce any chance of epithelial ovarian cells being seeded into the peritoneum. All surgeries before 2003 were full abdominal hysterectomies, including BSO. After 2003 many women have opted for laparoscopic BSO.

Women in the control group were those on annual screening with ovarian ultrasound and serum CA125. The protocol being used since 2000 is for screening women above 35 years of age. However, since 1990, some women were being screened from age 30 years. Follow-up was from first scan to time of most recent scan, cancer detection or death. Patients were treated according to preference and not randomised to surveillance or surgery. All ovarian cancers relevant to the individual's risk were confirmed from hospital records, death certificates or from the NWCIS.

\section{Results \\ (1) BSO group}

A total of 300 women have undergone risk-reducing surgery with 265 full hysterectomies and BSO, and 35 laparoscopic BSO surgical procedures. A total of 160 $B R C A 1 / 2$ carriers have undergone surgery (104 BRCA1, 56 $B R C A 2)$. Three occult malignancies were discovered at the time of surgery. Two BRCA1 carriers had a stage 3 and a stage $1 \mathrm{c}$ cancer, and a $B R C A 2$ carrier had a stage $1 \mathrm{~b}$ cancer. The stage 3 cancer resulted in the death of the patient within 3 years. A fourth BRCA1 mutation carrier had disseminated abdominal malignancy from her original primary breast cancer.

There have been 2400.37 years of follow-up (range 0-27 years; mean 8.17 years median 7.27$)$ and $15.79(95 \%$ confidence interval (CI) 8.3-24.6) expected cancers. No peritoneal cancers have occurred. The maximum number of cancers that would be consistent at $P=0.05$, with observing zero among the surgical patients, is nine. A total of 14 women developed breast cancer in the follow-up period (68 mutation carriers had also undergone risk-reducing mastectomy).

\section{Control group}

There were 503 women who have not chosen risk-reducing BSO. A further 135 patients who eventually undertook riskreducing BSO contributed to the follow-up time up until their surgery (two had occult cancers at BSO). There were 3444.25 years follow-up (range $1-17$ years; mean 6.8 years; median 7.18, 94 women > 10 years), 15.93 (95\% CI $8.4-$ 24.75) cancers were expected and 19 were found (15 excluding two cancers detected on prevalence screen and two at risk-reducing BSO). A total of 180 women were from families with known BRCA1 or BRCA2 mutations (20 were at $50 \%$ risk of a mutation). A total of 104 BRCA1 mutation carriers had 655.41 years follow-up with 8.19 expected cancers and 56 BRCA2 carriers had 316.14 years follow-up with 1.58 expected cancers. Nine cancers occurred in $B R C A 1$ mutation carriers, one in a BRCA2 carrier and two in carriers of HNPCC-related mismatch repair gene defects. These two women aged 32 and 34 years, with MSH2 mutations were detected at stage $1 \mathrm{c}$ at incident screens (endometrioid $2653 \mathrm{C}>\mathrm{T}$ (Q885X) mutation; and clear cell with a $942+3 \mathrm{~A}>\mathrm{T}$ splicing mutation). Furthermore, one patient with an earlier bilateral breast cancer who developed ovarian cancer in follow-up had a BRCA1 5313-12 G>A mutation, which could affect splicing. Two patients aged 32 and 73 years, a BRCA1 and a BRCA2 mutation carrier, 
Table 1 Breakdown of women and cancer per risk category and choice

\begin{tabular}{|c|c|c|c|c|}
\hline & $\begin{array}{l}\text { Number } \\
\text { total }\end{array}$ & $\begin{array}{l}\text { Number BSO } \\
\text { (cancers) }\end{array}$ & $\begin{array}{l}\text { Number contributing to } \\
\text { screening, but choosing } B S O^{a}\end{array}$ & $\begin{array}{c}\text { Number screened not } \\
\text { choosing surgery (cancers) }\end{array}$ \\
\hline BRCA1 & 208 & $104(2)$ & $45(1)^{b}$ & $104(9)$ \\
\hline BRCA2 & 112 & $56(1)$ & $18(1)^{b}$ & $56(1)$ \\
\hline HNPCC-proven MMR mutation & 13 & 1 & 1 & $12(2)$ \\
\hline Family history lifetime risk $10 \%+$ & 273 & 88 & $51(0)$ & $203(2)$ \\
\hline Family history lifetime risk $<10 \%$ & 80 & 24 & $7(0)$ & $56(1)^{c}$ \\
\hline Tested negative family BRCA1/2 mutation & 70 & $18(0)$ & $7(0)$ & $52(0)$ \\
\hline Not tested for family BRCA1/2 mutation & 47 & $9(0)$ & $6(0)$ & $20(0)$ \\
\hline Total & 803 & 300 & 135 & 503 \\
\hline
\end{tabular}

${ }^{a}$ These women contributed time to the screening data, but eventually chose BSO before symptoms or abnormalities on screening.

${ }^{b}$ Chose BSO surgery while on ovarian screening, but no abnormality on screening tests.

'Borderline mucinous tumour.

The higher rates of expected cancers in the BSO group reflect the longer follow-up of BRCA1/2 mutation carriers in the surgical group.

developed occult stage 1 ovarian cancer detected at riskreducing surgery 6 and 10 months after normal ultrasound and CA125 screening (see above). All other cancers occurred between 46 and 68 years of age. Three cancers, including a borderline malignancy, occurred in individuals untested for BRCA $1 / 2$. The majority of screened individuals who did not develop ovarian cancer were also unscreened for BRCA1/2 (Table 1). A total of 6 of the 17 women (19 including the two found at BSO) with ovarian cancer in the screened group have died, and five with stage 3 disease have relapsed after chemotherapy and have residual disease. It is likely, therefore, that 11 of 17 (65\%) will eventually succumb to ovarian cancer compared with 1 of 15.8 (7\%) of the expected cancers in the surgery group.

Peritoneal primary rates in women not having undergone BSO Of 245 ovarian cancers in BRCA1/2 mutation carriers on the database, only seven (3\%) were designated as peritoneal primary malignancy.

\section{Discussion}

This report documents the medium term follow-up of 300 women at high-risk of ovarian cancer who have undergone risk-reducing surgery. This study has reinforced the efficacy of this surgery to reduce ovarian cancer risk. No cancers have occurred after surgery, whereas over 15 were expected, and the upper end of the $95 \%$ CI are still equivalent to an $81 \%$ reduction in risk. This is significantly better than the pooled data from 10 studies, which gave a 79\% overall reduction. ${ }^{16}$ Two peritoneal primaries were reported in a large combined series of 259 BRCA1/2 mutation carriers undertaking BSO, although 56 ovarian cancers occurred in 292 controls. ${ }^{17}$ This was equivalent to a $96 \%$ reduction in risk. In a smaller study of 170 women, 98 chose risk-reducing salpingo-oophorectomy, and peritoneal cancer was diagnosed in one woman. ${ }^{18}$ Amongst 72 women who chose surveillance in this study, ovarian cancer occurred in four, and peritoneal cancer in one; this was equivalent to a $75 \%$ reduction in risk. Although our report has fewer mutation carriers than the first of these reports from 2002, ${ }^{17}$ it also includes women from families without proven mutations. Three of the expected cancers and six of those in the controls occurred in women not known to be BRCA1/2 mutation carriers. A larger combined study from the first two groups on 1079 mutation carriers found an $85 \%$ reduction in gynaecological malignancy for BRCA1 carriers. ${ }^{18}$ Three peritoneal primary cancers occurred in a mean follow-up of 40 months amongst 325 women undertaking surgery.

The potential accuracy of our risk prediction is shown by the control group, although the CIs were still wide. The cancers detected in $B R C A 1 / 2$ carriers and in non-carriers were virtually identical to that predicted. The control group, although larger, contained a substantial group of women with lower overall predicted risk (Table 1). This accounts for the $0.46 \%$ annual risk compared with the $0.66 \%$ risk predicted in the surgery group. Although we have not carried out a direct matching exercise of cases and controls, the accuracy of the predictions has been bourne out by our analysis.

The rate of occult malignancy is identical to that found in the two earlier reports in mutation carriers. ${ }^{17,19}$ A total of 3 of 160 (2\%) had occult malignancy in our report compared with 6 of $259(2.3 \%)$ in the larger report. ${ }^{17} \mathrm{~A}$ higher rate of occult malignancy has been found if extra pathological examinations are carried out with seven occult malignancies discovered, four in the fallopian tube and three in the ovaries, amongst 76 cases. ${ }^{20} \mathrm{Six}$ of these were microscopic. It is arguable whether we would have detected more cancers with a more thorough protocol, but none of the patients without malignancy detected have gone on to develop signs of invasive disease.

In contrast to the study by Rebbeck et al, ${ }^{17}$ which used controls who were not necessarily undergoing surveillance, we have only used controls who were prospectively followed on an active annual surveillance programme. Despite this surveillance, women on an annual programme of CA125 and ovarian ultrasound will still present 
Table 2 Comparison of the oophorectomy and control groups for ovarian and breast cancer incidence and death

\begin{tabular}{|c|c|c|}
\hline & Oophorectomy group & Screened group \\
\hline Number & 300 & 503 \\
\hline Number BRCA1/2 carriers & 160 & 160 \\
\hline Number MMR carriers & 1 & 12 \\
\hline Ovarian cancer in follow-up & 0 & 15 \\
\hline Stage of ovarian cancers & - & 2 in stage 1,3 in stage 2,9 in stage 3,1 in stage 4 \\
\hline Ovarian cancer death & 1 & 6 \\
\hline Breast cancer death (diagnosed after start date) ${ }^{a}$ & $7(1)^{a}$ & $6(1)^{a}$ \\
\hline (6) Other non-breast/ovarian cancer deaths & 0 & 4 \\
\hline Breast cancer diagnosed after start date & 14 & 13 \\
\hline Breast cancer diagnosed before start date & 66 & 56 \\
\hline
\end{tabular}

Abbreviation: MMR, mismatch repair mutation carriers.

${ }^{a}$ Only one breast cancer death per group was attributable to a breast cancer occurring after commencement of follow-up in either BSO or screening group.

predominantly with late-stage disease (Table 2). This is shown from our own data and a combined analysis from five centres of 63 ovarian tumours in a screening programme with 10 -year survival of only $36 \% .^{21}$ As such, 11 ovarian cancer deaths may have been expected out of the 15.79 cancers expected in the surgical group, with one actually dying from a cancer detected at surgery. As such, close to 13 ovarian cancers and 10 ovarian cancer deaths will most likely have already been prevented in the surgery group. With further follow-up, it is likely that this benefit would be greatly increased given the absence of cancers found after surgery. We are not able to comment on any benefit from the prevention of breast cancer or breast cancer death due to the absence of direct matching of cases in this study. It is nonetheless likely that breast cancers will be prevented in women undergoing early BSO with around a $50 \%$ reduction in risk. ${ }^{17}$

The debate as to the frequency of peritoneal malignancy after surgery still pertains. In 324 women who underwent prophylactic BSO in an ovarian cancer family registry, primary peritoneal carcinoma histologically indistinguishable from primary ovarian adenocarcinoma developed in six of them. ${ }^{20}$ Such malignancy could reflect undetected occult disease removed at surgery or the development of disease in ovarian epithelial tissue lodged during embryological development. In particular, the high rate of tubal malignancy and occult disease in reports ${ }^{22}$ emphasises the need to remove the fallopian tube in its entirety. Alternatively, epithelial ovarian cells on the surface of the ovaries could be displaced into the peritoneum at the time of surgery. We are aware of one peritoneal cancer occurring in a 56-year-old BRCA1 carrier 4 years after BSO at another centre in our region. An increased risk in the peritoneum is suggested by the apparently higher rates of peritoneal primary disease in mutation carriers after surgery. Only 6 of $244(2.4 \%)$ of ovarian cancers were designated as of peritoneal origin in our mutation carriers, excluding the case found after surgery from another centre. It is possible that some stage 3 or 4 disease is wrongly labelled as starting in the ovaries, nonetheless, the risk reduction of $96 \%$ in the paper by Rebbeck et $a l^{17}$ suggests that $<10 \%$ of the ovarian type risk resides in the peritoneum. Absolute risks of peritoneal cancer in follow-up are still $<1 \%$ in BSO series. ${ }^{17,19}$ The result that no peritoneal cancers were found during follow-up after surgery, may be because of the relatively small number of patients or the length of followup. Nonetheless, our data support the careful approach to ovarian risk-reducing surgery carried out by this and many other centres, although direct comparisons of techniques in large numbers of women would be required for proof of benefit.

\section{References}

1 Young RC, Walton LA, Ellenberg SS et al: Adjuvant therapy in stage I and stage II epithelial ovarian cancers. Results of two prospective randomised trials. N Engl J Med 1990; 322: 1021-1027.

2 Evans DGR: Risk estimation in ovarian cancer; in Lalloo F, Kerr B, Friedman J, Evans DGR (eds): Risk Assessment and Management in Cancer Genetics. Oxford University Press, Oxford, 2005, pp 73-82.

3 Chappius PO, Foulkes WD: Overview of the clinical genetics of ovarian cancer; in Morrison PJ, Hodgson SV, Haites NE (eds): Familial Breast and Ovarian Cancer: Genetics, Screening and Management. Cambridge University Press, Cambridge, 2002, pp 43-72.

4 Stratton JF, Pharoah P, Smith SK, Easton D, Ponder BA: A systematic review and meta-analysis of family history and risk associated with ovarian cancer. Br J Obstet Gynaecol 1998; 105: 493-499.

5 Risch HA, McLaughlin JR, Cole DE et al: Population BRCA1 and BRCA2 mutation frequencies and cancer penetrances: a kincohort study in Ontario, Canada. J Natl Cancer Inst 2006; 98: $1694-1706$.

6 Thompson D, Easton DF: Cancer Incidence in BRCA1 mutation carriers. J Natl Cancer Inst 2002; 94: 1358-1365.

7 Moller P, Hagen AI, Apold J et al: Genetic epidemiology of BRCA mutation-family history identifies less than $50 \%$ of mutation carriers. Eur J Cancer 2007; 43: 1713-1717.

8 Ford D, Easton DF, Stratton M et al: Genetic heterogeneity and penetrance analysis of the BRCA1 and BRCA2 genes in breast cancer families. The Breast Cancer Linkage Consortium. Am J Hum Genet 1998; 62: 676-689. 
9 Aarnio M, Sankila R, Pukkala E et al: Cancer risk in mutation carriers of DNA-mismatch-repair genes. Int J Cancer 1999; 81: 214-218.

10 Hogg R, Freidlander M: Biology of epithelial ovarian cancer: implications for screening women at high genetic risk. I Clin Oncol 2004; 22: 1315-1327.

11 Hermsen BB, Olivier RI, Verheijen RH et al: No efficacy of annual gynaecological screening in $B R C A 1 / 2$ mutation carriers; an observational follow-up study. Br J Cancer 2007; 96: 1335-1342.

12 Gaarenstroom KN, van der Hiel B, Tollenaar RA et al: Efficacy of screening women at high risk of hereditary ovarian cancer: results of an 11-year cohort study. Int J Gynecol Cancer 2006; 16 (Suppl 1): $54-59$.

13 Dorum A, Heimdal K, Lovslett $\mathrm{K}$ et al: Prospectively detected cancer in familial breast/ovarian cancer screening. Acta Obstet Gynecol Scand 1999; 78: 906-911.

14 Stirling D, Evans DG, Pichert G et al: Familial ovarian cancer screening: current protocols are ineffective in detecting early stage ovarian malignancy. J Clin Oncol 2005; 23: 5588-5596.

15 Munkarah A, Chatterjee M, Tainsky MA: Update on ovarian cancer screening. Curr Opin Obstet Gynecol 2007; 19: 22-26.

16 Rebbeck TR, Kauff ND, Domchek SM: Meta-analysis of risk reduction estimates associated with risk-reducing salpingo- oophorectomy in BRCA1 or BRCA2 mutation carriers. I Natl Cancer Inst 2009; 101: 80-87.

17 Rebbeck TR, Lynch HT, Neuhausen SL et al: Prevention and observation of surgical end points study group. Prophylactic oophorectomy in carriers of BRCA1 or BRCA2 mutations. $N$ Engl J Med 2002; 346: 1616-1621.

18 Kauff ND, Domchek SM, Friebel TM et al: Risk-reducing salpingooophorectomy for the prevention of BRCA1- and BRCA2associated breast and gynecologic cancer: a multicenter, prospective study. J Clin Oncol 2008; 26: 1331-1337.

19 Kauff ND, Satagopan JM, Robson ME et al: Risk-reducing salpingo-oophorectomy in women with a BRCA1 or BRCA2 mutation. N Engl J Med 2002; 346: 1609-1615.

20 Piver MS, Jishi MF, Tsukada Y, Nava G: Primary peritoneal carcinoma after prophylactic oophorectomy in women with a family history of ovarian cancer. A report of the Gilda Radner Family Ovarian Cancer Registry. Cancer 1993; 71: 2751-2755.

21 Evans DG, Gaarenstroom KN, Stirling D et al: Screening for familial ovarian cancer: poor survival of BRCA1/2 related cancers. J Med Genet 2008 [E-pub ahead of print].

22 Powell CB, Kenley E, Chen LM et al: Risk-reducing salpingooophorectomy in BRCA mutation carriers: role of serial sectioning in the detection of occult malignancy. J Clin Oncol 2005; 23: 127-132. 